\title{
Filter for TV and video cameras
}

\author{
L.I. Berezhinsky ${ }^{1}$, Dae-Yong Park ${ }^{2}$, Chang-Min Sung ${ }^{2}$, Kwang-Ho Kwon ${ }^{3}$, and Sang-Hoon Chai ${ }^{4}$ \\ 1 Institute of Semiconductor Physics, NAS of Ukraine, 45, prospect Nauky, 03028 Kyiv, Ukraine \\ Phone: +38 (044) 525-5778. E-mail: lib1938@yahoo.com \\ 2 Havit Information Co., 59-3 Jang-Dong, Yusong-gu, Daejeon, 305-343, Korea \\ E-mail: bspark@havit.co.kr \\ 3 Department of Electronic Engineering, Hanseo University, 360, Daegok-Ri, Haemi-Myun, Seosan, Choongnam, \\ 356-820, Korea.E-mail: khkwon@creneed.com \\ 4 Department of Electronic Engineering, Hoseo University, 21, Sechul-Ri, Baebang-Myun, Asan, Choongnam, 336- \\ 795, Korea
}

\begin{abstract}
An infrared-cut filter for TV and video cameras was calculated and fabricated. The filter contains 28 alternating $\mathrm{SiO}_{2}$ and $\mathrm{TiO}_{2}$ layers. The filter was calculated using the principle of unequal-thickness layers. This filter has a transmittance of $95 \%$ in 400 to $650 \mathrm{~nm}$ range and $1 \%$ in 700 to1150 nm range.
\end{abstract}

Keywords: interference coating, multilayer interference filter, cut filter.

Manuscript received 14.01.05; accepted for publication 18.05.05.

It is necessary to use infrared-cut filters to create a highquality image using TV and video cameras in natural (solar) light. This necessity is caused by the following fact. Human eyes perceive light within the spectral range 400 to $700 \mathrm{~nm}$. However, the photosensitivity of a photodetector (as a rule, is a silicon-target matrix used in TV and video cameras reaches up to $1200 \mathrm{~nm}$. Therefore, the presence of a photocurrent produced by 700 to $1200 \mathrm{~nm}$ waves in a total electric signal is undesirable because it does not carry any useful information about image colors. This allows to formulate the following requirements for optical properties of filters: the transmittance in 400 to $650 \mathrm{~nm}$ range should be not less than $90-95 \%$ and no more than $1 \%$ in 700 to1200 nm range.

In [1], it was reported about the filter provided the transmittance of more than $90 \%$ in 400 to $700 \mathrm{~nm}$ range and less than $1 \%$ in 700 to $930 \mathrm{~nm}$ one. At the same time, this filter has the very high transmittance (80 $90 \%$ ) in the region of 1000 to $1200 \mathrm{~nm}$. It seems that the role of this transmittance can be ignored because the photosensitivity of silicon in this region is very small. However, a simple calculation shows that it is not so. The solar radiation power in 1000 to $1200 \mathrm{~nm}$ range near the Earth surface contains from 18 to $20 \%$ of the solar radiation power in the whole visible region of 400 to $650 \mathrm{~nm}$. Therefore, the contribution into a total photocurrent from 1000 to $1200 \mathrm{~nm}$ waves can be appreciable despite the small photosensitivity of silicon in this region. In this article, we describe a filter with the transmittance of about $95 \%$ within the region of 400 to $650 \mathrm{~nm}$ and its improved characteristics in the region of 1000 to $1200 \mathrm{~nm}$.

The filter represents the system $\left(n_{\mathrm{H}} n_{\mathrm{L}}\right){ }^{N} n_{\mathrm{H}}$ of alternating layers with high- and low-refractive indexes and optical thickness $n d=\lambda / 4(\lambda$ - characteristic wavelength, $d$ - layer thickness). As $n_{\mathrm{L}}$ material we used $\mathrm{SiO}_{2}$ with the refractive index $n_{\mathrm{L}}=1.45454$, and as $n_{\mathrm{H}}$ material we used $\mathrm{TiO}_{2}$ with $n_{\mathrm{H}}=2.25565$. According to M. Born and E. Wolf [2], the reflection of a multilayer system reaches $99 \%$ at $N=6$, i.e., a filter must contain 13 alternating layers $n_{\mathrm{H}}, n_{\mathrm{L}}, n_{\mathrm{H}}, n_{\mathrm{L}}, \ldots, n_{\mathrm{H}}$.

A band where the filter has a very high reflection is called the suppression band, designated as $R_{100}$. The wavelength corresponding to the middle of $R_{100}$ band is designated as $\lambda_{0}$ (reference wavelength). The layers have the optical thickness equal to $\lambda_{0} / 4$.

The half-width of $R_{100}$ band in the units $g=\lambda_{0} / \lambda$ depends only on the difference between refractive indexes $n_{\mathrm{H}}$ and $n_{\mathrm{L}}$ and is defined by the following expression [3]:

$\Delta g=\frac{2}{\pi} \arcsin \frac{n_{\mathrm{H}}-n_{\mathrm{L}}}{n_{\mathrm{H}}+n_{\mathrm{L}}}$.

Let us define the width of $R_{100}$ band for our data. The high reflection region of our filter should extend from 700 to $1200 \mathrm{~nm}$. The center of this range lies at $\lambda_{0}=950 \mathrm{~nm}$. Substituting $n_{\mathrm{H}}=2.25565$ and $n_{\mathrm{L}}=1.45454$ into (1) we get $\Delta g \approx 0.13$. $R_{100}$ bandwidth has the value of $\lambda_{0} \cdot 2 \Delta g=247 \mathrm{~nm}$. Thus, our materials $\mathrm{SiO}_{2}$ and $\mathrm{TiO}_{2}$ can supply only $247 \mathrm{~nm} R_{100}$ bandwidth, while the filter bandwidth should have its extent of $1200-700=500 \mathrm{~nm}$. It means that to construct a filter with $500 \mathrm{~nm} R_{100}$ bandwidth it is necessary to use two (or three) systems of layers with different reference wavelengths $\lambda_{0}{ }^{\prime}$ and $\lambda_{0}{ }^{\prime \prime}$. The wavelengths $\lambda_{0}{ }^{\prime}$ and $\lambda_{0}{ }^{\prime \prime}$ are chosen from the requirement that the bands of high 
reflection partly overlap. It is clear that the formation of $R_{100}$ band with the width of $500 \mathrm{~nm}$ by means of twolayer systems with different $\lambda_{0}{ }^{\prime}$ and $\lambda_{0}$ " using $n_{\mathrm{SiO}_{2}}$ and $n_{\mathrm{TiO}_{2}}$ is a very difficult task. At the same time, the usage of three-layer systems makes the filter design very complex, and its practical realization is very difficult.

We have constructed a filter of two systems such as $(\mathrm{HL})^{6} \mathrm{H}$ with $\lambda_{0}{ }^{\prime}=736 \mathrm{~nm}$ and $\lambda_{0}{ }^{\prime \prime}=900 \mathrm{~nm}$ and achieved further widening $R_{100}$ band by means of suppression (increasing) of lateral transmittance minima. We obtained the suppression band from 680 to $1050 \mathrm{~nm}$ with transmittance of 1 to $1.5 \%$. However, a large number of lateral transmittance minima arises on the shortwave side of this band in the region of 400 to $650 \mathrm{~nm}$ and on the longwave one of $1050 \mathrm{~nm}$. The lateral minima in the region of 400 to $650 \mathrm{~nm}$ are generated by the layer system at $\lambda_{0}{ }^{\prime}=736 \mathrm{~nm}$, and the ones with wavelengths longer than $1050 \mathrm{~nm}$ are generated by the layer system at $\lambda_{0} "=900 \mathrm{~nm}$. The number of these lateral minima is equal to the number of layers in every system, and their depths reach $60 \%$. Hence, the problem of a filter construction lies in elimination of these minima and preservation of the transmittance in the range of 400 to $600 \mathrm{~nm}$ at the level of $95 \%$ and vice versa increasing the depth of transmittance minima down to zero in the region of 1050 to1200 $\mathrm{nm}$.

There are a few receptions to correct the transmittance on both sides of $R_{100}$ band. One of them is to use layers with unequal optical thicknesses according the condition

$n_{\mathrm{H}} h_{\mathrm{H}}+n_{\mathrm{L}} h_{\mathrm{L}}=\lambda_{0} / 2$,

where $h_{\mathrm{H}}$ and $h_{\mathrm{L}}$ are geometrical thicknesses of layers with a high- and low-refractive indexes. The transmittance is increased in a shortwave range of $R_{100}$ band and simultaneously is decreased in a longwave range when $n_{\mathrm{H}} h_{\mathrm{H}} / n_{\mathrm{L}} h_{\mathrm{L}}>1$. The relation $n_{\mathrm{H}} h_{\mathrm{H}} / n_{\mathrm{L}} h_{\mathrm{L}}<1$ gives the opposite result.

A very effective method for increasing the transmittance of a lateral minimum is creation of the last $n_{\mathrm{L}}$ layer at the optical thickness $\lambda_{0} / 8$ on the surface of this multilayer system. However, it should be noted that the use of such layer as well as introduction of matching layers between the substrate and multilayer system does not allow to obtain the transmittance more than $80 \%$ in 400 to $420 \mathrm{~nm}$ range.

The range of 400 to $420 \mathrm{~nm}$ is shortwave in relation to $R_{100}$ band with the center at $\lambda_{0}{ }^{\prime}=736 \mathrm{~nm}$. However, it can be considered as the longwave one in relation to $R_{100}$ band with the center at $\lambda_{01}$, which satisfies the condition

$n_{\mathrm{H}} h_{\mathrm{H}}=n_{\mathrm{L}} h_{\mathrm{L}}=(3 / 4) \lambda_{01}$

or

(3/4) $\lambda_{01}=(1 / 4) \lambda_{0}^{\prime} ; \lambda_{01}<\lambda_{0}^{\prime}$.

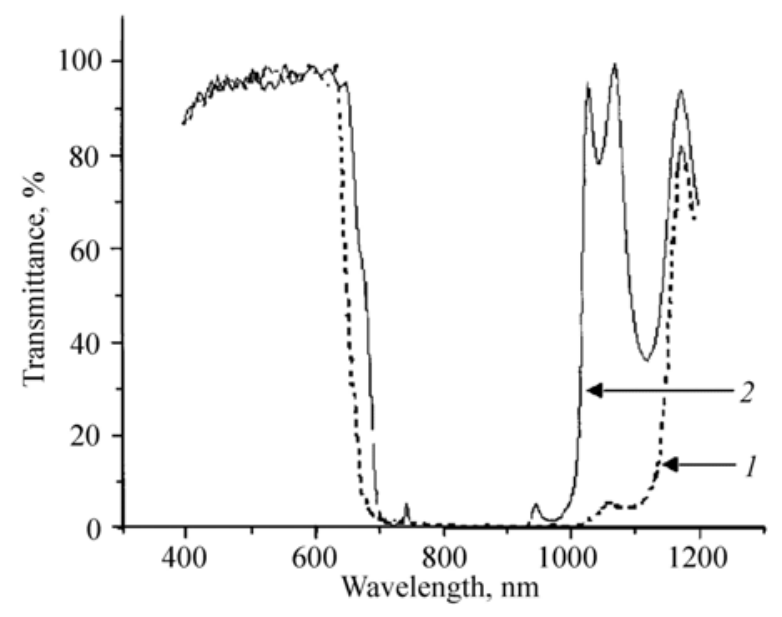

Fig. 1. Optical characteristic of IR-cut filter (simulation): $1-$ this work, 2 - filter from [1].

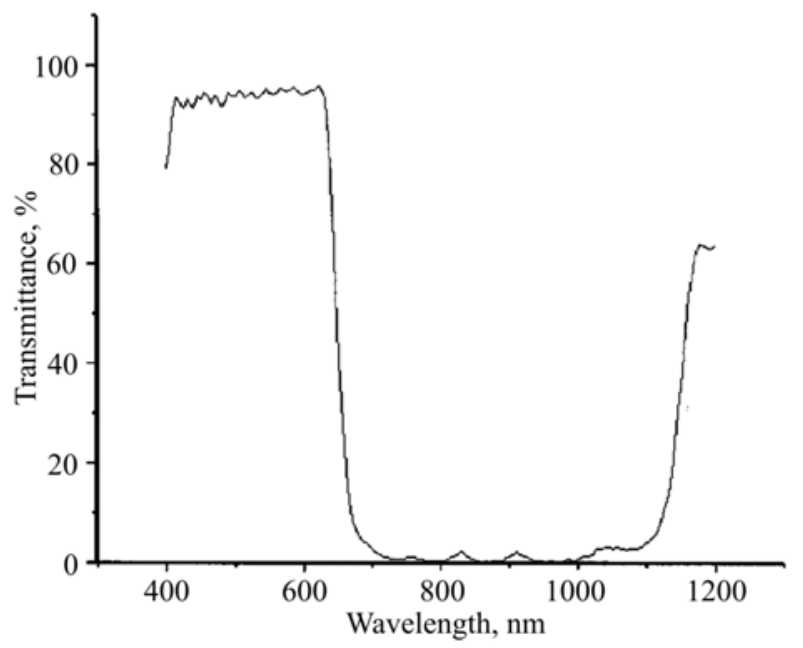

Fig. 2. Optical characteristic of the real IR-cut filter (experiment).

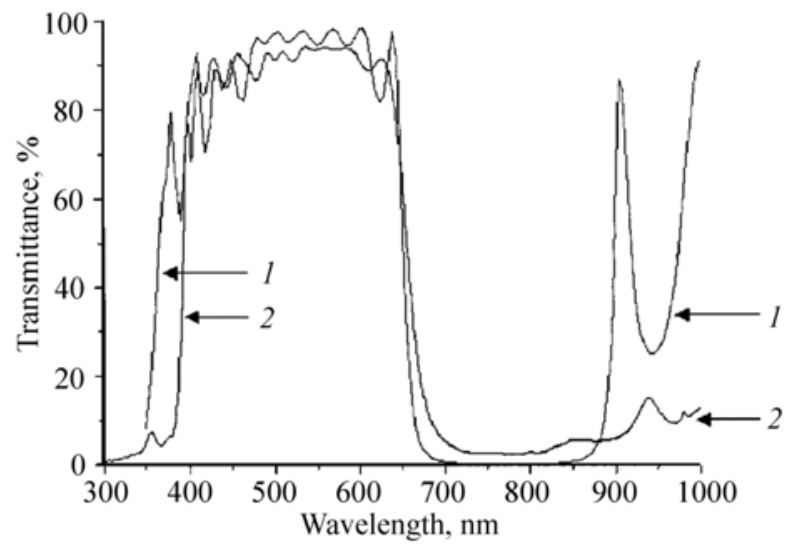

Fig. 3. Optical characteristics of IR-cut filters, produced by Melles Griot (USA) (1) and Taeyoung (Korea) (2). 
The design and layer thicknesses of the IR cut-filter.

\begin{tabular}{|c|c|c|c|}
\hline Layer & Material & Refractive index & Optical thickness (in $\lambda_{0}$ ) \\
\hline 1 & $\mathrm{SiO}_{2}$ & 1.45454 & 0.170 \\
\hline 2 & $\mathrm{TiO}_{2}$ & 2.25565 & 0.332 \\
\hline 3 & $\mathrm{SiO}_{2}$ & 1.45454 & 0.335 \\
\hline 4 & $\mathrm{TiO}_{2}$ & 2.25565 & 0.329 \\
\hline 5 & $\mathrm{SiO}_{2}$ & 1.45454 & 0.332 \\
\hline 6 & $\mathrm{TiO}_{2}$ & 2.25565 & 0.325 \\
\hline 7 & $\mathrm{SiO}_{2}$ & 1.45454 & 0.331 \\
\hline 8 & $\mathrm{TiO}_{2}$ & 2.25565 & 0.316 \\
\hline 9 & $\mathrm{SiO}_{2}$ & 1.45454 & 0.330 \\
\hline 10 & $\mathrm{TiO}_{2}$ & 2.25565 & 0.316 \\
\hline 11 & $\mathrm{SiO}_{2}$ & 1.45454 & 0.330 \\
\hline 12 & $\mathrm{TiO}_{2}$ & 2.25565 & 0.316 \\
\hline 13 & $\mathrm{SiO}_{2}$ & 1.45454 & 0.330 \\
\hline 14 & $\mathrm{TiO}_{2}$ & 2.25565 & 0.316 \\
\hline 15 & $\mathrm{SiO}_{2}$ & 1.45454 & 0.295 \\
\hline 16 & $\mathrm{TiO}_{2}$ & 2.25565 & 0.250 \\
\hline 17 & $\mathrm{SiO}_{2}$ & 1.45454 & 0.270 \\
\hline 18 & $\mathrm{TiO}_{2}$ & 2.25565 & 0.250 \\
\hline 19 & $\mathrm{SiO}_{2}$ & 1.45454 & 0.270 \\
\hline 20 & $\mathrm{TiO}_{2}$ & 2.25565 & 0.250 \\
\hline 21 & $\mathrm{SiO}_{2}$ & 1.45454 & 0.265 \\
\hline 22 & $\mathrm{TiO}_{2}$ & 2.25565 & 0.235 \\
\hline 23 & $\mathrm{SiO}_{2}$ & 1.45454 & 0.270 \\
\hline 24 & $\mathrm{TiO}_{2}$ & 2.25565 & 0.240 \\
\hline 25 & $\mathrm{SiO}_{2}$ & 1.45454 & 0.275 \\
\hline 26 & $\mathrm{TiO}_{2}$ & 2.25565 & 0.235 \\
\hline 27 & $\mathrm{SiO}_{2}$ & 1.45454 & 0.285 \\
\hline 28 & $\mathrm{TiO}_{2}$ & 2.25565 & 0.285 \\
\hline Substrate & Glass & 1.51218 & \\
\hline
\end{tabular}

It means that we can consider a band with the center $\lambda_{01}$ as a band of the next order concerning the band with the center $\lambda_{0}{ }^{\prime}$. Therefore, if we change the transmittance of the filter in the longwave range for $\lambda_{0}{ }^{\prime}$ band, we simultaneously in the same way change the transmittance in the longwave range for $\lambda_{01}$ band, i.e., in $400-420 \mathrm{~nm}$ range. On this basis, we have used layers of an unequal optical thickness for increasing the transmittance in the longwave range of $\lambda_{0}{ }^{\prime}=736 \mathrm{~nm}$. Choosing the layer geometrical thickness we used the relationship

$\alpha \cdot n_{\mathrm{H}} h_{\mathrm{H}}+(2-\alpha) n_{\mathrm{L}} h_{\mathrm{L}}=\lambda_{0}{ }^{\prime} / 2$,

where the coefficient $\alpha<1$ and $n_{\mathrm{H}} h_{\mathrm{H}}=n_{\mathrm{L}} h_{\mathrm{L}}=\lambda_{0}{ }^{\prime} / 4$. For optimizing the value of $\alpha$, we used the geometrical thicknesses $h_{\mathrm{H}}{ }^{\prime}$ and $h_{\mathrm{L}}{ }^{\prime}$ with the relation $n_{\mathrm{L}} h_{\mathrm{L}}{ }^{\prime} / n_{\mathrm{H}} h_{\mathrm{H}}{ }^{\prime}=1.2$. The transmittance of 90 to $95 \%$ in the range 400 to $420 \mathrm{~nm}$ was obtained for these layer thicknesses. However, the transmittance in the range of 550 to $650 \mathrm{~nm}$ was decreased. We have tried to improve the reduction of transmittance in the range of 500 to $650 \mathrm{~nm}$ introducing the additional and matching layers. The deposition of the latter layer with $\lambda_{0}{ }^{\prime} / 8$ optical thickness allowed to increase the transmittance in this range up to $80 \%$. Further magnification was achieved by the introduction of the matching $\lambda_{0}{ }^{\prime} / 4$ layer between the substrate and multilayer system.

The refractive index of the matching layer can be found from the relationship [4]

$n=\sqrt{N_{E} n_{S}}$,

where $N_{E}$ is the effective refractive index of the multilayer system, and $n_{S}$ is the refractive index of the substrate. It may happen so that a substance with refractive index $n$ is nonexistent in nature. In this case, it is possible to construct system of three layers such as $(a \mathrm{H} b \mathrm{~L} a \mathrm{H})$ that will play functionally the same role as well as a quarter-wave layer with refractive index $n$. Coefficients $a$ and $b$ can be determined by the method of effective layers [5]. For our case, the refractive index of the matching layer and its design was calculated in [1].

The filter was constructed on the basis of two-layer systems with the reference waves $\lambda_{0}{ }^{\prime}=736 \mathrm{~nm}$ and $\lambda_{0}{ }^{\prime \prime}=900 \mathrm{~nm}$. After the combination of these systems on the substrate, the optimization of the layer thickness at the interface of these systems was carried out using a 
computer. The final filter contains 28 layers and has the structure $(0.44 \mathrm{~L}, 0.88 \mathrm{H}, 0.44 \mathrm{~L}) 0.5 \mathrm{~L}(\mathrm{HL})^{12} \mathrm{H} 0.5 \mathrm{~L}$. The layer thicknesses are summarized in Table. The transmittance of the calculated filter and filter from [1] is shown in Fig. 1. It is seen that, in the visible range, the transmittance is nearly the same for both filters and reaches $95 \%$ in 450 to $620 \mathrm{~nm}$ range. The transmittance of the calculated filter averages about 2 to $3 \%$ higher than that of the filter in [1]. In the infrared region, however, the distinction of transmittance is drastic. The calculated filter has the transmittance close to zero in the region from 700 to $1030 \mathrm{~nm}, 5 \%$ - in the region of 1050 to $1120 \mathrm{~nm}$, and, only for $1150-1200 \mathrm{~nm}$, the transmittance band of more than $80 \%$ is observed.

The transmittance of the obtained filter manufactured using the above-mentioned design is shown in Fig. 2. The small $(\sim 3 \%)$ transmittance reduction of the real filter as compared with the simulated one is caused by monitoring errors for layer thicknesses during manufacturing. The accuracy of the latter was $\pm 2 \mathrm{~nm}$. For comparison, the transmittance of available commercial filters comprising 29 to 30 layers and manufactured by Taeyoung (Korea) and Melles Griot (USA), which are used in modern TV and video cameras, are shown in Fig. 3.

\section{References}

1. L. Berezhinsky, K.H. Kwon, B.S. Park, Infrared-cut filter // Jpn J. Appl. Phys. 40, N 10, p. 5953-5954 (2001).

2. M. Born and E. Wolf, Principles of optics. Pergamon Press, Oxford, 2nd ed., p. 51 (1964).

3. T.N. Nikolayeva, Interference coverings, Mashinostroyeniye, Leningrad (1973) (in Russian) .

4. H.A. Macleod, Thin-film optical-filters. Adam Hilger, Bristol, p. 73 (1986).

5. L.I. Epstein, The design of optical filters // J. Opt. Soc. Amer. 12, N 11, p. 806-810 (1952). 